\title{
Demonstration of the exponential decay law using beer froth
}

\author{
A Leike \\ Ludwig-Maximilians-Universität, Sektion Physik, Theresienstr. 37, D-80333 München, Germany \\ E-mail: leike@theorie.physik.uni-muenchen.de
}

Received 22 June 2001, in final form 8 October 2001

Published 17 December 2001

Online at stacks.iop.org/EJP/23/21

\begin{abstract}
The volume of beer froth decays exponentially with time. This property is used to demonstrate the exponential decay law in the classroom. The decay constant depends on the type of beer and can be used to differentiate between different beers. The analysis shows in a transparent way the techniques of data analysis commonly used in science - consistency checks of theoretical models with the data, parameter estimation and determination of confidence intervals.
\end{abstract}

Exponential laws are common to many physical phenomena. Examples are the amplitude of an oscillator subject to linear friction, the discharge of a capacitor, cooling processes or radioactive decays. The demonstration described here has the advantages that it is cheap, clear and motivating because it investigates an everyday phenomenon. It can easily be repeated by the students elsewhere.

The decay of beer froth is mentioned as a very short notice in [1]. It is described in several German textbooks of mathematics. Recently, it also attracted the attention of Bavarian pupils [2].

The data analysis proposed in this paper has much in common with real science-see, for example, the determination of the Higgs mass by the LEP collaborations [3]. The techniques involved are of great practical importance but are often poorly understood by students [4].

Exponential decay can be demonstrated using beer froth, the volume of which reduces exponentially with time [1]. The exponential law can readily be derived from the assumption that the volume of froth $\mathrm{d} V$ disappearing in the time between $t$ and $t+\mathrm{d} t$ is proportional to the volume $V$ present at the time $t, \mathrm{~d} V=-(V / \tau) \mathrm{d} t$. In a cylindrical beer mug with an area $A$, the volume is proportional to the height, $\mathrm{d} V=A \mathrm{~d} h$. The phenomenological theory of exponential decay predicts the height as a function of time

$$
h^{t h}(t)=h(0) \exp \left(-\frac{t}{\tau}\right)
$$

The constant $\tau$ is a free parameter of the theory. It defines how fast the froth decays; during the time $\tau$ the amount $1-1 / \mathrm{e} \approx 63 \%$ of the froth disappears. Different kinds of beer have, in general, different parameters $\tau$. 
Table 1. The data $h^{\exp }\left(t_{i}\right)$ and their errors $h^{\exp }\left(t_{i}\right)$ for three different beers. The last line contains the best estimates for $\tau$ together with their errors of $68 \%$ (95\%) confidence.

\begin{tabular}{|c|c|c|c|c|c|c|}
\hline \multirow[b]{2}{*}{$t(s)$} & \multicolumn{2}{|c|}{ Erdinger Weissbier } & \multicolumn{2}{|c|}{ Augustinerbräu München } & \multicolumn{2}{|c|}{ Budweiser Budvar } \\
\hline & $h^{\exp } \mathrm{cm}^{-1}$ & $\Delta h^{\exp } \mathrm{cm}^{-1}$ & $h^{\exp } \mathrm{cm}^{-1}$ & $\Delta h^{\exp } \mathrm{cm}^{-1}$ & $h^{\exp } \mathrm{cm}^{-1}$ & $\Delta h^{\exp } \mathrm{cm}^{-1}$ \\
\hline 0 & 17.0 & 0.0 & 14.0 & 0.0 & 14.0 & 0.0 \\
\hline 15 & 16.1 & 0.3 & 11.8 & 0.3 & 12.1 & 0.4 \\
\hline 30 & 14.9 & 0.4 & 10.5 & 0.3 & 10.9 & 0.4 \\
\hline 45 & 14.0 & 0.4 & 9.3 & 0.5 & 10.0 & 0.4 \\
\hline 60 & 13.2 & 0.4 & 8.5 & 0.6 & 9.3 & 0.4 \\
\hline 75 & 12.5 & 0.6 & 7.7 & 0.6 & 8.6 & 0.4 \\
\hline 90 & 11.9 & 0.4 & 7.1 & 0.7 & 8.0 & 0.3 \\
\hline 105 & 11.2 & 0.4 & 6.5 & 0.8 & 7.5 & 0.3 \\
\hline 120 & 10.7 & 0.4 & 6.0 & 0.8 & 7.0 & 0.3 \\
\hline 150 & 9.7 & 0.4 & 5.3 & 1.1 & 6.2 & 0.3 \\
\hline 180 & 8.9 & 0.3 & 4.4 & 1.2 & 5.5 & 0.4 \\
\hline 210 & 8.3 & 0.4 & 3.5 & 0.9 & 4.5 & 0.4 \\
\hline 240 & 7.5 & 0.4 & 2.9 & 1.1 & 3.5 & 0.5 \\
\hline 300 & 6.3 & 0.5 & 1.3 & 0.7 & 2.0 & 0.5 \\
\hline 360 & 5.2 & 0.5 & 0.7 & 0.5 & 0.9 & 0.4 \\
\hline$\chi_{\min }^{2}$ & \multicolumn{2}{|c|}{11.1} & \multicolumn{2}{|c|}{12.5} & \multicolumn{2}{|c|}{24.4} \\
\hline$\tau_{\text {best }} \pm \Delta \tau \mathrm{s}^{-1}$ & \multicolumn{2}{|c|}{$276 \pm 7( \pm 14)$} & \multicolumn{2}{|c|}{$124 \pm 6( \pm 12)$} & \multicolumn{2}{|c|}{$168 \pm 4( \pm 8)$} \\
\hline
\end{tabular}

In the following, the demonstration is described in detail. In our experiment, a cylindrical beer mug with a diameter of $7.2 \mathrm{~cm}$ was filled with beer immediately after opening the bottle. The temperature of the beer was $19^{\circ} \mathrm{C}$.

The froth appears while filling the mug with the beer. The froth reaches its maximum height within a few seconds. This indicates that the typical time scale of the expansion of the froth is a few seconds. On the other hand, the froth lasts for a few minutes (see table 1). Therefore, the time scale for the decay is a few minutes. The two time scales are very different. We therefore assume that a few seconds after the time where the froth reaches its maximum height only the decay plays a significant role.

We began with the measurement at the time where the froth dropped to a certain initial height $h^{\exp }(0)=h(0)$. The error $\Delta h^{\exp }(0)$ in the measurement of $h(0)$ was estimated to be $2 \mathrm{~mm}$.

We investigated three different beers. With every beer, the experiment was repeated several times. We performed seven experiments with Erdinger Weissbier (the author's favourite!), four experiments with Augustinerbräu München and four experiments with Budweiser Budvar. Our data are shown in table 1 . The entries for $h^{\exp }\left(t_{i}\right)$ are obtained by averaging over all individual measurements at time $t_{i}$. To obtain the errors $\Delta h^{\exp }\left(t_{i}\right)$ of the measurements $h^{\exp }\left(t_{i}\right)$, we first calculated the root of the variance of all individual measurements at the time $t_{i}$. The relative error of $h(0)$ was added quadratically to the resulting relative error.

The error $\Delta t_{i}$ of our time measurements was below $1 \mathrm{~s} . \Delta t_{i}$ can be translated into an error in the height $\Delta h\left(t_{i}\right)$ using equation (1)

$$
|\Delta h(t)|=\left|\frac{\partial h(t)}{\partial t}\right| \Delta t=\frac{\Delta t}{\tau} h(t) .
$$

Having in mind the values for $\tau$ obtained in our experiment, the impact of $\Delta t_{i}$ on the error in the height is considerably smaller then the error $\Delta h^{\exp }\left(t_{i}\right)$ from the direct measurement. We therefore neglect the error $\Delta t_{i}$ in the time measurement.

With the experimental data in table 1 and the phenomenological theory (1), one can calculate 


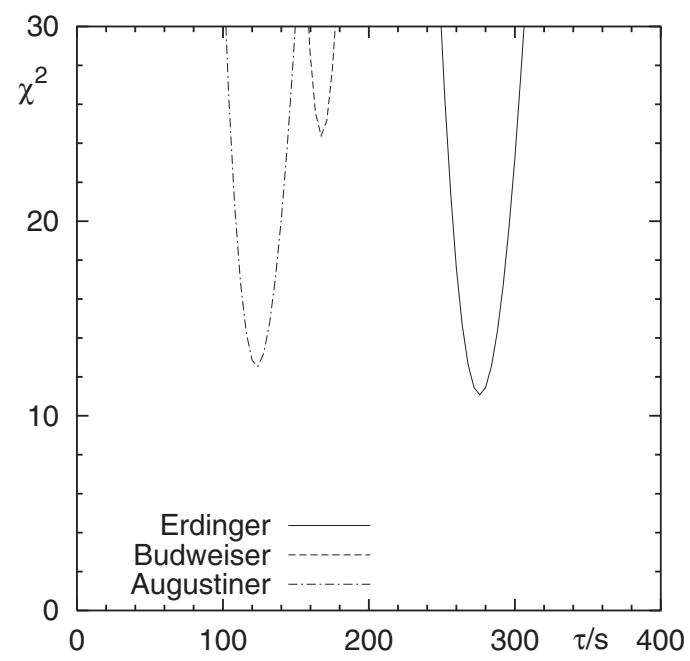

Figure 1. $\chi^{2}$ as function of $\tau$ for different kinds of beer.

$$
\chi^{2}=\sum_{i=1}^{n} \frac{\left[h^{\exp }\left(t_{i}\right)-h^{t h}\left(t_{i}\right)\right]^{2}}{\left[\Delta h^{\exp }\left(t_{i}\right)\right]^{2}}
$$

Figure 1 shows $\chi^{2}$ as a function of $\tau$. This fit has $N=13$ degrees of freedom because we have $n=15$ data points and two constraints. The first constraint is the fixed $h(0)$, the second constraint is the parameter $\tau$, which is defined from the data. The minimum values for $\chi^{2}$ as a function of $\tau$ for the three different beers are 11.1, 12.5 and 24.4 for Erdinger Weissbier, Augustinerbräu München and Budweiser Budvar respectively. They are given in table 1 .

If the data point errors $h^{\exp }\left(t_{i}\right)-h^{\text {th }}\left(t_{i}\right)$ are Gaussian, then the minimum of $\chi^{2}$ is distributed as a $\chi^{2}$ random variable. The minimum of $\chi^{2}$ is a measure of the goodness of the fit. The $\chi^{2}$ of a good fit with $N$ degrees of freedom should be approximately in the range $N \pm \sqrt{2 N}$. (See the appendix for some information about $\chi^{2}$ and [5] for a short review on probability and statistics.) The $\chi^{2}$ for Erdinger and Augustinerbräu indicate a good fit (see table 1). The $\chi^{2}$ for Budweiser is larger. The probability of getting a $\chi^{2}$ of 24.4 or larger with 13 degrees of freedom is about $3 \%$. However, the probability of getting such a large $\chi^{2}$ in at least one of the three independent fits is $9 \%$. We conclude that the theory of exponential decay is consistent with our experimental data. Then the determination of the model parameter $\tau$ using the data makes sense. The best fits to the parameter $\tau$ correspond to $\chi_{\min }^{2}$, the minimum $\chi^{2}$. We will denote it as $\tau_{\text {best }}$. Figure 2 shows the experimental data together with the best fits.

Under the condition that the experimental data are consistent with the theory, confidence intervals for the theoretical parameters can be defined. The decay law (1) depends only on one parameter $\tau$. A confidence interval $\left(\tau_{-}, \tau_{+}\right)$with a probability of $x \%$ means that the true value of the parameter $\tau$ is between $\tau_{-}$and $\tau_{+}$with a probability of $x \%$. Confidence intervals are found by exploring the properties of $\chi^{2}$ near its minimum $\chi_{\min }^{2}$ (see for example [5]). In particular, confidence intervals of $\tau$ with a probability of $68 \%(95 \%)$ are found by the condition $\chi^{2}=\chi_{\min }^{2}+1$ (4) (see also the appendix). The corresponding values $\tau_{ \pm}=\tau_{\text {best }} \pm \Delta \tau$ are given in the last row of table 1.

Our results suggest that the three kinds of beer may be distinguished by the lifetime of the froth only. However, before one can make a definite statement, one has to study carefully the dependence of $\tau_{\text {best }}$ on the temperature and on the date of production of the beer. These extensive studies are left to the reader. The author did only one preliminary measurement with Erdinger Weissbier at a temperature of $11^{\circ} \mathrm{C}$ and found $\tau=255 \pm 14 \mathrm{~s}$ (one standard 


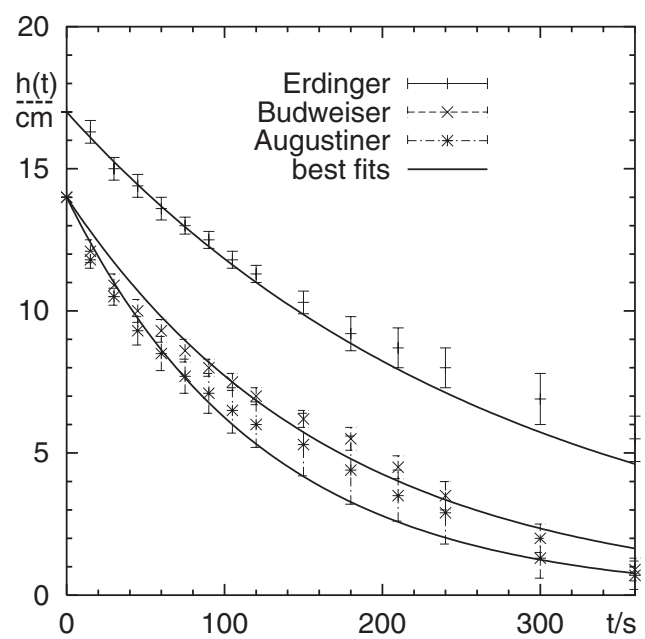

Figure 2. The height of the froth for different kinds of beer as function of time. Shown are the data and the best fits.

deviation, i.e. $68 \%$ confidence). This indicates that there is no substantial dependence of $\tau_{\text {best }}$ on the temperature.

An approximate determination of $\tau$ in the exponential decay (1) is possible with pocket calculators through a linear regression to $\ln (h(t) / h(0))$. It is equivalent to finding the minimum of the expression

$$
\sum_{i=1}^{n}\left(\ln \frac{h^{\exp }\left(t_{i}\right)}{h(0)}+\frac{t}{\tau}\right)^{2} .
$$

The results for $\tau$ are 290, 134 and 155 s for Erdinger Weissbier, Augustinerbräu and Budweiser respectively. These values are several standard deviations away from the values obtained in the $\chi^{2}$ fit. One of the reasons for this discrepancy originates in the simple procedure (4), which ignores the different experimental errors $\Delta h^{\exp }\left(t_{i}\right)$ in the data. The resulting values for $\tau$ can therefore only be a rough estimate.

Alternatively, one could test a model of linear decay of the froth

$$
h^{t h}(t)=h(0)\left(1-\frac{t}{T}\right) \theta(T-t)
$$

where $T$ is the time in which all the froth decays. The Heaviside function $\theta(T-t)$ ensures that the height cannot become negative. One can test the linear model by calculating $\chi^{2}$ according to formula (3). The minima of $\chi^{2}$ are 129, 86 and 161. The probability of having a $\chi^{2}$ of 86 or larger in a $\chi^{2}$ distribution with 13 degrees of freedom is about $10^{-12}$. This is the probability that, under the condition that the model of linear decay is correct, the observed data arose due to a statistical fluctuation. This probability is very small indicating that the model of linear decay is inconsistent with the experimental data for all three beers. Therefore, the linear theory should be rejected and a determination of the parameter $T$ from the data is meaningless.

The proposed demonstration is simple and motivating. It explains scientific methods using an everyday phenomenon. It can be included in undergraduate courses to show how to prove quantitatively the consistency of a phenomenological law with experimental data. The next step towards a theory, not discussed here, would be a deviation of the phenomenological law from first principles. 


\section{Acknowledgment}

I would like to thank L B Okun for encouraging me to write this paper.

\section{Appendix}

To make the paper self-consistent, the formulae regarding the goodness of a fit used in the main part of the paper are derived here. See [5] and references therein for more details.

Let us assume that we have $N$ Gaussian distributed random variables $x_{i}, i=1, \ldots, N$ with mean 0 and variance 1 . Consider the sum

$$
\chi^{2}=\sum_{i=1}^{N} x_{i}^{2}
$$

The random variable $\chi^{2}$ has $N$ degrees of freedom. It corresponds to the $\chi^{2}$ function given in (3), which also consists of a sum of the square of Gaussian random variables with mean 0 and variance 1 .

We are interested in the distribution of the random variable $\chi^{2}$. The distribution of the square of one Gaussian distributed variable can easily be obtained from the Gaussian distribution

$$
\begin{aligned}
\frac{2}{\sqrt{2 \pi}} \exp \left(-\frac{x^{2}}{2}\right) \mathrm{d} x & =\frac{1}{x \sqrt{2 \pi}} \exp \left(-\frac{x^{2}}{2}\right) \mathrm{d} x^{2} \\
& =\frac{1}{\sqrt{z} \sqrt{2 \pi}} \exp \left(-\frac{z}{2}\right) \mathrm{d} z=\chi_{1}^{2}(z) \mathrm{d} z, \quad z \geqslant 0 .
\end{aligned}
$$

The function $\chi_{1}^{2}(z)$ is called the $\chi^{2}$ distribution for one degree of freedom.

The characteristic function $\Phi(u)$ associated with a distribution $f(z)$ is defined as

$$
\Phi(u) \equiv \int_{-\infty}^{\infty} \exp (\mathrm{i} u z) f(z) \mathrm{d} z .
$$

The characteristic function of $\chi_{1}^{2}$ is

$$
\Phi_{1}(u)=\int_{0}^{\infty} \exp (\mathrm{i} u z) \chi_{1}^{2}(z) \mathrm{d} z=\frac{1}{(1-2 \mathrm{i} u)^{1 / 2}} .
$$

If $f_{1}\left(x_{1}\right)$ and $f_{2}\left(x_{2}\right)$ have characteristic functions $\Phi_{1}(u)$ and $\Phi_{2}(u)$, then the characteristic function of the distribution of the random variable $c_{1} x_{1}+c_{2} x_{2}$ is

$$
\begin{aligned}
\Phi(u) & =\int_{-\infty}^{\infty} \mathrm{d} x_{1} \int_{-\infty}^{\infty} \mathrm{d} x_{2} \exp \left[\mathrm{i} u\left(c_{1} x_{1}+c_{2} x_{2}\right)\right] f\left(x_{1}\right) f\left(x_{2}\right) \\
& =\int_{-\infty}^{\infty} \mathrm{d} x_{1} \exp \left(\mathrm{i} u c_{1} x_{1}\right) f\left(x_{1}\right) \int_{-\infty}^{\infty} \mathrm{d} x_{2} \exp \left(\mathrm{i} u c_{2} x_{2}\right) f\left(x_{2}\right) \\
& =\Phi_{1}\left(c_{1} u\right) \cdot \Phi_{2}\left(c_{2} u\right) .
\end{aligned}
$$

The characteristic function of the sum of squares of $N$ independent Gaussian distributed variables is therefore

$$
\Phi_{N}(u)=\frac{1}{(1-2 \mathrm{i} u)^{N / 2}}
$$

The distribution associated with $\Phi_{N}(u)$ is obtained by the transformation inverse to (8)

$$
\chi_{N}^{2}(z)=\frac{1}{2 \pi} \int_{-\infty}^{\infty} \exp (-\mathrm{i} u z) \Phi_{N}(u) \mathrm{d} u=\frac{z^{N / 2-1} \mathrm{e}^{-z / 2}}{2^{N / 2} \Gamma(N / 2)}, \quad z \geqslant 0 .
$$


It is called the $\chi^{2}$ distribution for $N$ degrees of freedom. It has the mean $N$ and the variance $2 N$. The probability density (12) is used to estimate the goodness of a fit. The probability $P\left(z>\chi^{2}\right)$ that $z$ is larger than a given value $\chi^{2}$ is

$$
P\left(z>\chi^{2}\right)=\int_{\chi^{2}}^{\infty} \chi_{N}^{2}(z) \mathrm{d} z
$$

In particular, we get

$$
\int_{24.4}^{\infty} \chi_{13}^{2}(z) \mathrm{d} z \approx 0.03=3 \%
$$

which is the number quoted in the main text.

The probability $P\left(z<a^{2}\right)$ that $z$ is smaller than a given value $a^{2}$ is

$$
P(z<x)=\int_{0}^{a^{2}} \chi_{N}^{2}(z) \mathrm{d} z .
$$

In particular, we get for $N=1$ and $a^{2}=1$ or 4

$$
\int_{0}^{1} \chi_{1}^{2}(z) \mathrm{d} z \approx 0.68=68 \% \quad \text { or } \quad \int_{0}^{4} \chi_{1}^{2}(z) \mathrm{d} z \approx 0.95=95 \%
$$

which are the numbers quoted in the main text for the confidence intervals of $\tau$.

\section{References}

[1] Göbel R and Haubold K 1989 Freihandexperimente in Klasse 10 Phys. Schule 27/3 104 (in German)

[2] Newspaper announcement (in German) http://www.mainfranken.org/deutsch/jufo2001_presse2.html

[3] The LEP Collaborations, the LEP electroweak workung group and SLD heavy flavour and electroweak group, CERN-EP-200-021 webpage http://lepewwg.web.cern.ch/LEPEWWG/

Kawamoto T 2001 Electroweak data and standard model fit results 36th Rencontres de Moriond-2001 hepex/0105032

(Kawamoto T 2001 Preprint hep-ex/0105032)

[4] Earnshaw J C $1990 \chi^{2}$-or do the data fit the theory? Eur. J. Phys. 11338

[5] 2000 Review of particle properties Eur. Phys. J. C 151 